\title{
Clustering Temporal Gene Expression Data with Unequal Time Intervals
}

\author{
Luis Rueda \\ Department of Computer Science \\ University of Concepción, Edmundo Larenas 215 \\ Concepción, 4030000, Chile \\ Irueda@inf.udec.cl
}

\author{
Ataul Bari \\ School of Computer Science \\ University of Windsor, 401 Sunset Avenue \\ Windsor, ON, N9B 3P4, Canada \\ bari1@uwindsor.ca
}

\begin{abstract}
We have focused on the problem of clustering time-series gene expression data. We present a novel algorithm for clustering gene temporal expression profile microarray data, which is fairly simple but powerful enough to find an efficient distribution of genes over clusters. Using a variant of a clustering index can effectively decide upon the optimal number of clusters for a given dataset. The clustering method is based on a profile-alignment approach, which we propose and that minimizes the (square) area between two aligned vector profiles, to hierarchically cluster microarray time series data. The effectiveness of the proposed approach is demonstrated on two well-known, yeast and serum.
\end{abstract}

\section{Keywords}

Gene expression, clustering, time-series profiling

\section{INTRODUCTION}

Clustering genes based on the similarity of their temporal profile expressions is important for many studies, such as those genes that are functionally related or co-regulated [6]. Clustering gene expression data given in terms of time series is different from a general clustering problem, because exchanging two time points delivers quite different results, while it may not be biologically meaningful. Many unsupervised methods for gene clustering based on the similarity (or dissimilarity) of their microarray temporal profiles have been proposed in the past few years $[1,2,6,11]$. One of the methods for clustering microarray time-series data is based on a hidden phase model (similar to a hidden Markov model) to define the parameters of a mixture of normal distributions in a Bayesian-like manner, which are estimated by using expectation maximization [2]. Other methods based on correlation measures have been proposed for clustering genes using microarray time series data [3, 7]. The method proposed in [3] requires computing the mean expression levels of some candidate profiles using some pre-identified, arbitrarily

Permission to make digital or hard copies of all or part of this work for personal or classroom use is granted without fee provided that copies are not made or distributed for profit or commercial advantage and that copies bear this notice and the full citation on the first page. To copy otherwise, to republish, to post on servers or to redistribute to lists, requires prior specific permission and/or a fee.

Bionetics '07, December 10-13, 2007, Budapest, Hungary

Copyright 2007 ICST 978-963-9799-11-0. selected profiles. In [7], a method for clustering microarray time series data employing a jack-knife correlation coefficient with or without using the seeded candidate profiles is proposed. Specifying expression levels for the candidate profiles in advance for these correlation-based procedures requires estimating each candidate profile, which is made using a small sample of arbitrarily selected genes. This makes it vulnerable to the possibility of missing important genes, since the resulting clusters depend upon the initially chosen template genes.

Another method is to select and cluster genes using the ideas of order-restricted inference, where estimation makes use of known inequalities among parameters [10]. In this method, at first, potential candidate profiles of interest are defined and expressed in terms of inequalities between the expected gene expression levels at various time points. For a given candidate profile, the estimated mean expression level of each gene is computed and the best fitting profile for a given gene is selected using the goodness-of-fit criterion and the bootstrap test procedure. In this approach, two genes $\mathbf{x}_{1}$ and $\mathbf{x}_{2}$ fall into the same cluster if they show similar profiles in terms of directions of the changes of expression ratios (e.g. up-up-up-down-down), regardless how big/small is the change.

In [12], a minimum-square-error profile alignment approach to cluster microarray time series data was proposed. The idea is to pairwisely align two temporal profiles in such a way that the sum of square errors between two aligned vectors is minimized. The alignment procedure, however, does not consider the length of the interval between two time points at which individual measurements are taken.

In this paper, we propose a profile alignment approach to cluster temporal microarray data that minimizes the area between two aligned profiles. The hierarchical clustering algorithm uses a variant of a well-known clustering validity index that optimizes the number of clusters $[5,9]$. The profile alignment that we propose in this paper is different from that of [12] in the sense that: (i) the approach proposed in this paper considers unequal time intervals, which is usually the case in microarray time-series experiments, and (ii) the alignment is performed by minimizing the error between two continuous functions and not the "knot" points. Experiments on serum data and on pre-clustered yeast data show the effectiveness of the proposed method.

\section{AREA-BASED PROFILE ALIGNMENT}

Consider a dataset with $n$ samples $\mathcal{D}=\left\{\mathbf{x}_{1}, \mathbf{x}_{2}, \ldots, \mathbf{x}_{n}\right\}$, 
where $\mathbf{x}_{i}=\left[x_{i_{1}}, x_{i_{2}}, \ldots, x_{i_{m}}\right]^{t}$ is an $m$-dimensional feature vector that represents the expression ratio of gene $i$ at $m$ different time points, $\mathbf{t}=\left[t_{1}, t_{2}, \ldots, t_{m}\right]^{t}$. The aim is to partition $\mathcal{D}$ into $k$ disjoint subsets $\mathcal{D}_{1}, \mathcal{D}_{2}, \ldots, \mathcal{D}_{k}$, where $\mathcal{D}=\mathcal{D}_{1} \cup$ $\mathcal{D}_{2} \cup \ldots \cup \mathcal{D}_{k}$, and $\mathcal{D}_{i} \cap \mathcal{D}_{j}=\emptyset$, for $\forall i, j, i \neq j$, in such a way that a similarity (dissimilarity) cost function $\phi:\{0,1\}^{n \times k} \rightarrow$ $\Re$ is maximized (minimized).

We propose an efficient alignment algorithm that takes two features vectors, and produces two new vectors in such a way that the area between "aligned" vectors is minimized. The idea is described in Figure 1. In Figure 1(a) two vectors are shown before alignment. Figure 1(b) shows the "aligned" vectors such that the area between the profiles is minimized, i.e. they were aligned in such a way that the total area covered by the triangle $\{u, v, z\}$ and the polygon $\{z, w, q, e, r, k, h, g, s\}$ is minimized.

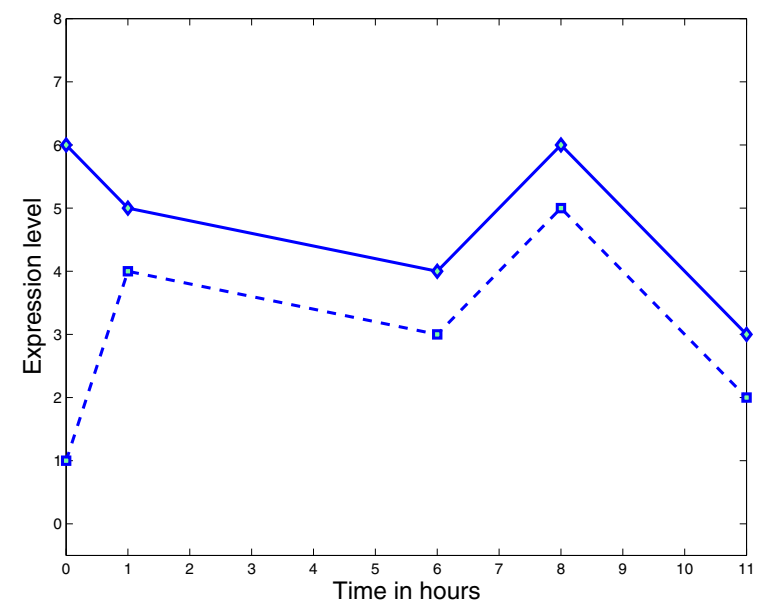

(a)

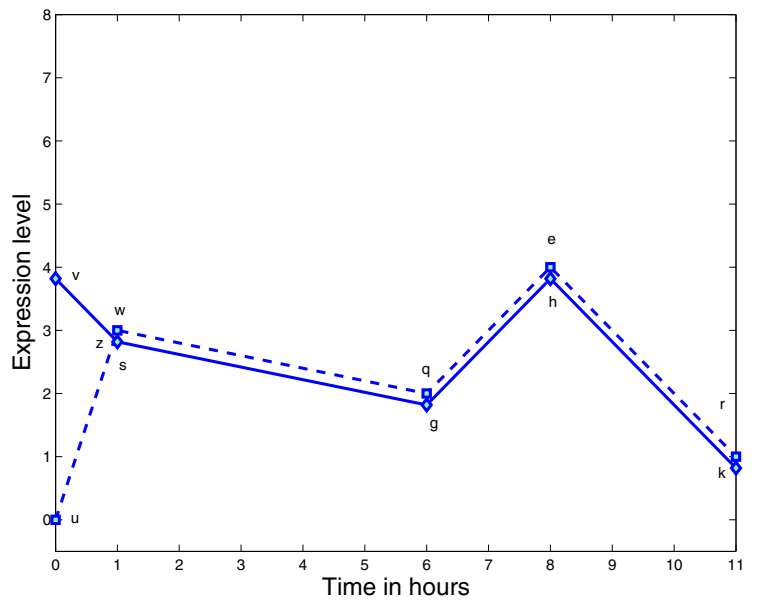

(b)

Figure 1: (a) Two unaligned profiles. (b) The two "aligned" profiles obtained after applying (1) - (4) such that the area between each pair of lines is minimized.

Let, $\mathbf{t}=\left[t_{1}, t_{2}, \ldots, t_{m}\right]^{t}$ be the vector representing the time points, and the two profiles, $\mathbf{x}=\left[x_{1}, x_{2}, \ldots, x_{m}\right]^{t}$, and $\mathbf{y}=$ $\left[y_{1}, y_{2}, \ldots, y_{m}\right]^{t}$ be two profiles, whose expression ratios were measured at time points given in $\mathbf{t}$, which are to be aligned.
The aim is to find a scalar $a$ that minimizes the total area between the two profiles, e.g., between the lines that join the expression ratios. To do this, we first "slide" down $\mathbf{x}$ and obtain a new vector, $\mathbf{x}^{\prime}$, as follows:

$$
\mathbf{x}^{\prime}=\left[x_{1}^{\prime}, x_{2}^{\prime}, \ldots, x_{m}^{\prime}\right]^{t} \leftarrow \mathbf{x}-x_{1} .
$$

Now, assume that the straight line that joins points $\left(t_{i-1}, x_{i-1}^{\prime}\right)$ and $\left(t_{i}, x_{i}^{\prime}\right)$ is given by $x_{i-1}^{\prime}+\frac{\left(x_{i}^{\prime}-x_{i-1}^{\prime}\right)}{t_{i}-t_{i-1}} u$, and for points $\left(t_{i-1}, y_{i-1}\right)$ and $\left(t_{i}, y_{i}\right)$ is given by $y_{i-1}-a+$ $\frac{\left(y_{i}-y_{i-1}\right)}{t_{i}-t_{i-1}} u$, where $u$ corresponds to the " $x$-axis". Let us use the following notation: $\hat{y}_{i}=y_{i}-y_{i-1}, \hat{x}_{i}^{\prime}=x_{i}^{\prime}-x_{i-1}^{\prime}$ and $\hat{t}_{i}=t_{i}-t_{i-1}$. Since we want to minimize the area between $\mathbf{x}$ and $\mathbf{y}$ (aligned), for all $t_{1}, t_{2}, \ldots, t_{m}$, we need to find $a$ that minimizes the sum of square errors between each pair of lines, equivalent to the following sum of integrals:

$$
f(a)=\sum_{i=2}^{m} \int_{t_{i-1}}^{t_{i}}\left[x_{i-1}^{\prime}+a-y_{i-1}+\frac{\hat{x}_{i}^{\prime}-\hat{y}_{i}}{\hat{t}_{i}} u\right]^{2} d u,
$$

by means of the first and second order conditions, resulting in:

$$
a=-\frac{\sum_{i=2}^{m}\left[\left(x_{i-1}^{\prime}-y_{i-1}\right) \hat{t}_{i}+\frac{\hat{x}_{i}^{\prime}-\hat{y}_{i}}{\hat{t}_{i}} \frac{\hat{t}_{i}^{2}}{2}\right]}{\sum_{i=2}^{m} \hat{t}_{i}}
$$

Then, a new vector, $\mathbf{y}^{\prime}$, is computed as follows:

$$
\mathbf{y}^{\prime}=\mathbf{y}-a .
$$

Let $f_{i}=\frac{\left(x_{i}^{\prime}-x_{i-1}^{\prime}\right)-\left(y_{i}^{\prime}-y_{i-1}^{\prime}\right)}{t_{i}-t_{i-1}}$. By computing the integrals, the distance between the two new vectors $\mathbf{x}^{\prime}$ and $\mathbf{y}^{\prime}$, $d\left(\mathbf{x}^{\prime}, \mathbf{y}^{\prime}\right)$, results in:

$$
\begin{aligned}
& d\left(\mathbf{x}^{\prime}, \mathbf{y}^{\prime}\right) \\
& =\sum_{i=2}^{m}\left(x_{i-1}^{\prime}-y_{i-1}^{\prime}\right)^{2} \hat{t}_{i}+\left(x_{i-1}^{\prime}-y_{i-1}^{\prime}\right) f_{i} \hat{t}_{i}^{2}+f_{i}^{2} \frac{\hat{t}_{i}^{3}}{3} .
\end{aligned}
$$

The second order condition is satisfied, i.e. $\frac{\partial^{2} f}{\partial^{2} a}=\frac{\partial}{\partial a} 2 \sum_{i=1}^{m}$ $\left(x_{1_{i}}-x_{2_{i}}+a\right)=2 m>0$. This indicates that a minimum is reached at $a=\frac{1}{m} \sum_{i=1}^{m}\left(x_{2_{i}}-x_{1_{i}}\right)$. Using (1) - (4) to obtain the value of $a$, we first align two profile vectors and then compute a distance function in the usual manner. It is not difficult to show that this alignment, used in conjunction with any metric $d$, is also a metric [12]. Note that once the alignment is applied, any metric $d$ can be used. In this paper, we have used the distance given in (5), since we meant to minimize the area between two aligned profiles.

The justification of the proposed alignment is depicted in Figure 2 with examples of temporal profiles for three pairs of genes. Figures 2(a), (b) and (c) show three pairs of genes without alignment. Using the Pearson correlation distance [10], genes in Figure 2(b) are most likely to be clustered together (as they produce the largest value for correlation coefficient among all three pairs of genes, which is 0.9053). Note that the prime interest is to cluster genes based on the variations of the expression ratios at the different time points. The aim is to find variations in terms of changes (or not) between different time points, independently of the "scale" in which the ratios lie. Then, genes from Figure 2(c) 
would be better candidates to be clustered together than the genes in Figs. 2(a) and (b). However, the value of the correlation coefficient between the pairs of genes in Figure 2 (c) is the minimum (0.8039) among all three pairs of genes. Figures 2 (d), (e) and (f) show the pairs of genes after aligning the genes from Figures 2(a), (b) and (c), respectively. A simple visual inspection shows that the genes in Figure $2(\mathrm{f})$ are closer to each other compared to the genes from the other two figures, Figures 3(d) and (e).

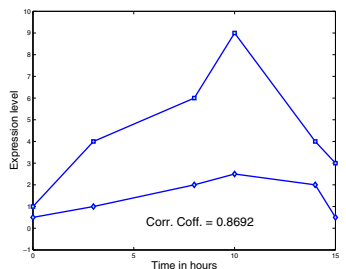

(a)

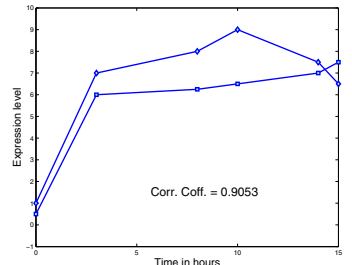

(b)

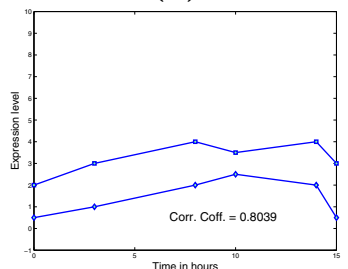

(c)

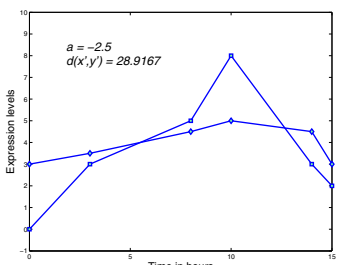

(d)

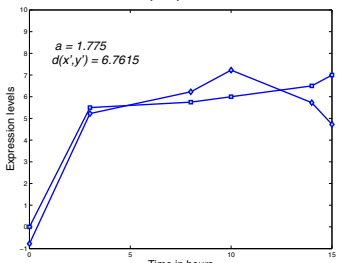

(e)

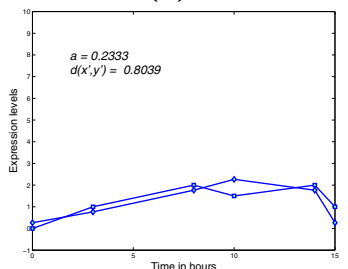

(f)
Figure 2: (a) Two genes that are likely to be clustered as in [10], although the difference among them in terms of rate of expression ratio changes between different time points is large. (b) Two genes with different profiles that are likely to be clustered together by correlation-based methods. (c) Two genes with similar profiles in terms of rate of expression ratio changes between different time points that may not be clustered together by the method proposed in [10] and the correlation-based methods. (d) Result after aligning the two genes from (a). (e) Result after aligning two genes from (b). (f) Result after aligning two genes from (c). After applying the proposed profile alignment, the differences between the genes in (d) and (e) are more notorious than in (f).

\section{THE CLUSTERING ALGORITHM}

Hierarchical agglomerative clustering is the method used in this paper. We apply complete linkage or furthest neighbors [4], which computes the distance between the furthest pair of points for each pair of clusters and merges the pair of clusters that has the minimum distance among all such distances between the pair of clusters under consideration. The generalized algorithm of hierarchical agglomerative clustering is slightly modified to obtain the desired number of clusters instead of a hierarchy of clusters, which is given in Algorithm 1, Agglomerative-Clustering.

The Agglomerative-Clustering algorithm receives two parameters as input, a complete microarray temporal dataset, $\mathcal{D}$, and the desired number of clusters $k$, and returns the dataset after partitioning it into $k$ clusters. The best number of clusters $k^{*}$ is chosen to maximize the $\mathcal{I}$-index as follows:

$$
\mathcal{I}(k)=\left(\frac{1}{k}\right)^{q} \times\left(\frac{E_{1}}{E_{k}} \times \mathcal{D}_{k}\right)^{p}
$$

where, $n$ is the total number of samples in the dataset, $E_{k}=$ $\sum_{i=1}^{k} \sum_{j=1}^{n} u_{i j} d\left(\mathbf{x}_{j}, \boldsymbol{\mu}_{i}\right), \mathcal{D}_{k}=\max _{i, j=1}^{k} d\left(\boldsymbol{\mu}_{i}, \boldsymbol{\mu}_{j}\right),\left\{u_{i j}\right\}_{k \times n}$ is the partition (or membership) matrix for the data, $\boldsymbol{\mu}_{i}$ is the center of cluster $\mathcal{D}_{i}, k$ is the number of clusters, and $d(.,$.$) is the distance computed as in (5).$

The decision rule is based on the furthest-neighbor distance between two clusters, which is computed using formula (5). The latter involves the alignment of each pair of profiles before applying a conventional (formula (5) in our case) distance function.

Algorithm Agglomerative-Clustering

Input: The dataset, $\mathcal{D}=\left\{\mathbf{x}_{1}, \mathbf{x}_{2}, \ldots, \mathbf{x}_{n}\right\}$, and $k$, the desired number of clusters.

Output: $k$ disjoint subsets $\mathcal{D}_{1}, \mathcal{D}_{2}, \ldots, \mathcal{D}_{k}$.

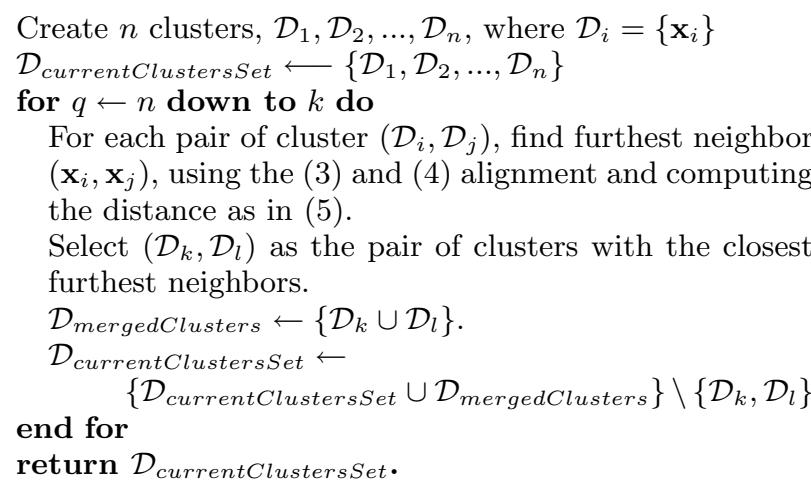

The partition matrix $\left\{u_{i j}\right\}$ is defined as a membership function such that $u_{i j}=1$, if $\mathbf{x}_{j}$ belongs to cluster $\mathcal{D}_{i}$, and zero otherwise. To compute the mean of cluster $\mathcal{D}_{i}, \boldsymbol{\mu}_{i}$, we follow a greedy approach that computes the mean and scatter for each cluster. It picks the first profile from the cluster and assigns it to be the current mean. Then, it picks the next gene profile from the cluster, applies the pairwise alignment with this profile to the current mean, and updates the current mean by taking the average of these two aligned profiles. The process continues until all the gene profiles are aligned and the current mean is updated correspondingly for each profile. Note that it is done in this way, otherwise it should be done like "multiple alignment", which is a nontrivial issue that remains open.

The cluster mean is used to compute the scatter of a cluster. Given a cluster $\mathcal{D}_{i}=\left\{\mathbf{x}_{i_{1}}, \mathbf{x}_{i_{2}}, \ldots, \mathbf{x}_{i_{n}}\right\}$, using (1) - (4) each $\mathbf{x}_{i_{j}}$ is aligned with the mean $\boldsymbol{\mu}_{i}$ producing a set of aligned profiles $\mathcal{D}_{i}^{\prime}=\left\{\mathbf{y}_{i_{1}}, \mathbf{y}_{i_{2}}, \ldots, \mathbf{y}_{i_{n}}\right\}$. The scatter is computed as $E_{i}=\sum_{j=1}^{i_{n}} d\left(\mathbf{y}_{i_{j}}, \boldsymbol{\mu}_{i}\right)$, where $d(.,$.$) is the distance$ computed as in (5).

\section{EXPERIMENTAL RESULTS}

We have tested the performance of our clustering method 
that performs profile alignment combined with agglomerative clustering (PAAC) on two well-known datasets, the serum [8] and yeast [3] datasets. For the serum dataset, we conducted experiments on a subset containing 517 out of 8,613 genes $^{1}$. We considered a range for potential numbers of clusters, which includes values of $k$ that lie between $\lceil\sqrt{n / 2}\rceil$ and $\lfloor\sqrt{3 n / 2}\rfloor$, i.e. $k=16$ to 27 . For each $k$, the $\mathcal{I}$-index was computed using values of $q$ from 0.3 to 1.0 . We have selected $q=0.7$, since we want to favor a large number of clusters, as $q=0.7<2.0=p$. The value 0.7 was found experimentally and confirmed with pre-clustered yeast data for which we found our method provides a high classification accuracy, as seen later. For $q=0.7$, the value of the index reaches to a maximum level when $k=21$. The plots are shown in Figure 4, where each plot represents a cluster. The $x$-axis in each plot represents the time in hours and the $y$-axis represents the expression ratio.

We have tested the results of our method with the results obtained by the Pearson correlation distance and the Spearman correlation distance methods. Clustering using the Pearson correlation distance is given in Figure 4. The comparison among the plots for PAAC and Pearson reveals the effectiveness of the method. For example, PAAC left clusters 1 to 5 containing a single gene each (IDs 328692, 470934, 361247,147050 and 310406, respectively). The Pearson correlation method, however, placed these genes in clusters 19 , 2, 2, 11 and 19, respectively. By visual inspection of all the temporal expression profiles, we noticed that these genes are differentially expressed and should be left alone in separate clusters, which is clearly done by PAAC. Also, PAAC produced four clusters containing only two profiles each, clusters 9 (IDs 356635 and 429460 ), 11 (IDs 26474 and 254436), 13 (IDs 280768 and 416842) and 16 (IDs 130476 and 130482). The Pearson correlation method clustered these genes as follows: 356635 and 429460 in cluster 16, 26474 and 254436 in cluster 21, 280768 and 416842 in cluster 2 and 130476 and 130482 in cluster 16 (Figure 4). Although the Pearson correlation method placed each pair of genes in the same cluster, it also placed some other genes with them. By looking at the plots of the profiles of the clusters produced by the Pearson correlation method and comparing them to the plots of the clusters of the corresponding genes produced by PAAC, it is clear that these pairs of genes are differentially expressed. For the Spearman correlation method, though non-linear, we observed that it outputs results comparable to those of the Pearson correlation, and is not able to identify and separate differentially expressed genes properly as good as PAAC (plots not shown).

In order to provide a biological significance of our results, we applied our method to a dataset containing the changes in gene expression during the cell cycle of the budding yeast, $S$. cerevisiae ${ }^{2}$ [13], in which expression ratios were measured at seventeen different time points, from $0 \mathrm{~min}$. to $160 \mathrm{~min}$. with an interval of 10 mins. The experiment monitored 6,220 transcripts for cell cycle-dependent periodicity and 221 functionally characterized genes with periodic fluctuation were listed in Table 1 of [13]. We applied PAAC to these 221 genes, using the same parameters as those for the serum dataset, obtaining the best number of clusters $k=28$. The clusters obtained using PAAC are shown in Figure 5. We

\footnotetext{
${ }^{1}$ http://genome-www.stanford.edu/serum/.

${ }^{2}$ http://genome-www.stanford.edu/cellcycle/data/rawdata/ individual.html.
}

also used the Pearson correlation coefficient to cluster the dataset (plots not shown). We observed that PAAC separates the genes by profiles in a wise manner, while the Pearson correlation method is not able to capture all variations in the time series.

Finally, we list in Tables 1-3, the 221 genes, where for each gene it is shown the cluster number PAAC assigns and the class (phase) that the gene is categorized as in Table 1 of [13]. An objective measure for comparing the two clusterings has been taken by computing the overall classification accuracy, which is computed as the number of genes that PAAC correctly assigned to one of the phases. The correct class (phase) is the one that PAAC assigns the largest number of genes. The overall classification accuracy was computed as the average of the individual accuracies for each cluster, resulting in $83.47 \%$, which is very high considering the fact that PAAC is an unsupervised classification algorithm.

\section{CONCLUSIONS}

We have proposed a method to cluster gene expression temporal profile microarray data. On two well-known reallife datasets, we have demonstrated that using hierarchical clustering with our method for similarity measure produced superior results when compared to that of the Pearson and Spearman correlation similarity measures.

We have applied a variant of the $\mathcal{I}$-index that can make a trade-off between minimizing the number of useful clusters and keeping the distinctness of individual clusters. We have also shown the biological significance of the results obtained by computing the classification accuracy of our method in pre-clustered yeast data - the accuracy was over $83 \%$.

PAAC can be used for effective clustering of gene expression temporal profile microarray data. Although we have shown the effectiveness of the method in microarray timeseries datasets, we are planning to investigate the effectiveness of the method as well in dose-response microarray datasets, and other time series microarray data.

\section{Acknowledgments:}

This research has been partially supported by the Chilean National Council for Technological and Scientific Research, FONDECYT grant No. 1060904.

\section{REFERENCES}

[1] A. Brazma and J. Vilo. Gene expression data analysis. FEBS Lett., 480:17-24, 2000.

[2] L. Bréhélin. Clustering Gene Expression Series with Prior Knowledge. In Lecture Notes in Computer Science, volume 3692, pages 27-38, October 2005.

[3] S. Chu, J. DeRisi, M. Eisen, J. Mulholland, D. Botstein, P. Brown, and I. Herskowitz. The transcriptional program of sporulation in budding yeast. Science, 282:699-705, 1998.

[4] S. Drăghici. Data Analysis Tools for DNA Microarrays. Chapman \& Hall, 2003.

[5] R. Duda, P. Hart, and D. Stork. Pattern Classification. John Wiley and Sons, Inc., New York, NY, 2nd edition, 2000.

[6] M. Eisen, P. Spellman, P. Brown, and D. Botstein. Cluster analysis and display of genome-wide expression patterns. In Proc. Natl Acad. Sci., volume 95, pages 14863-14868, USA, 1998. 

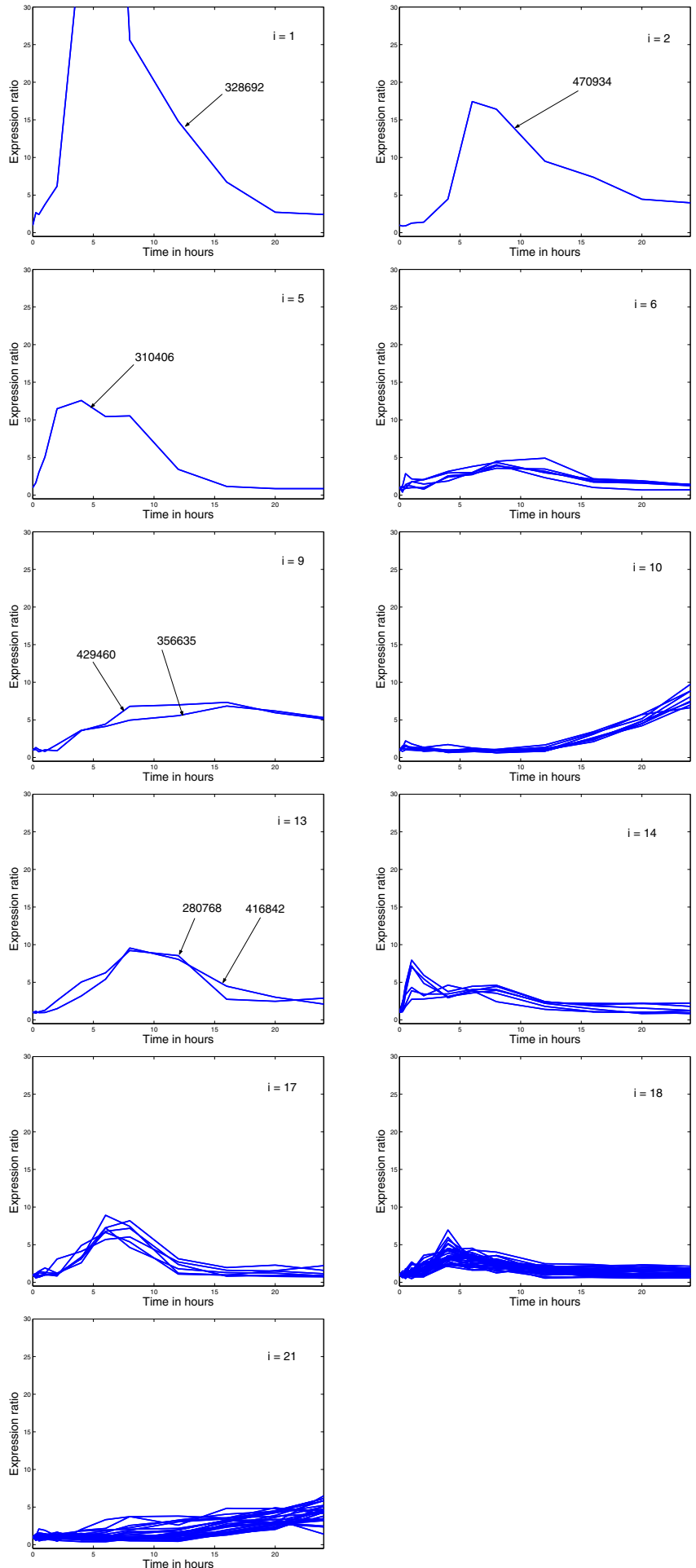

Time in hours

Time in hours
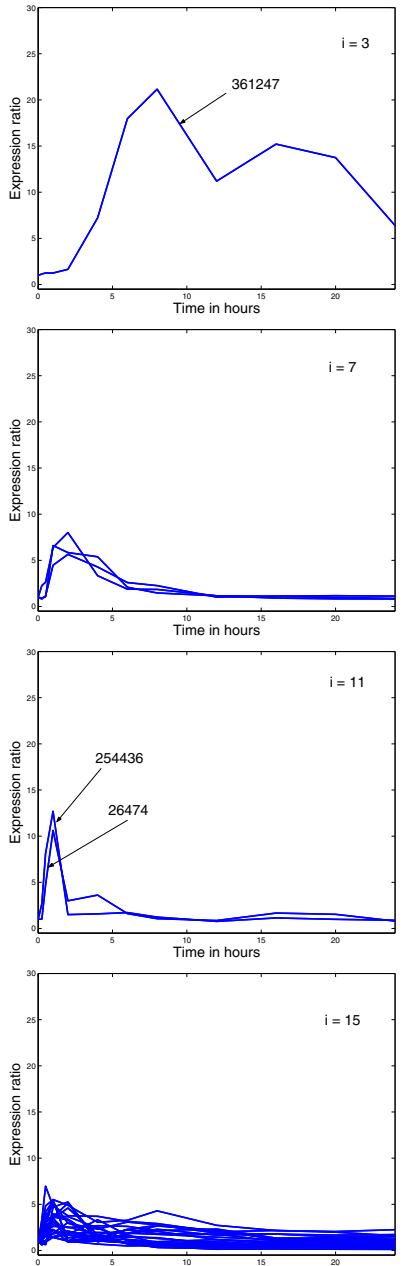

Time in hours

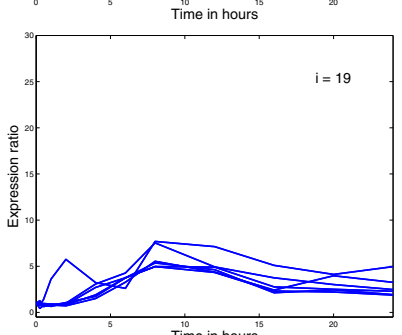

time in hours
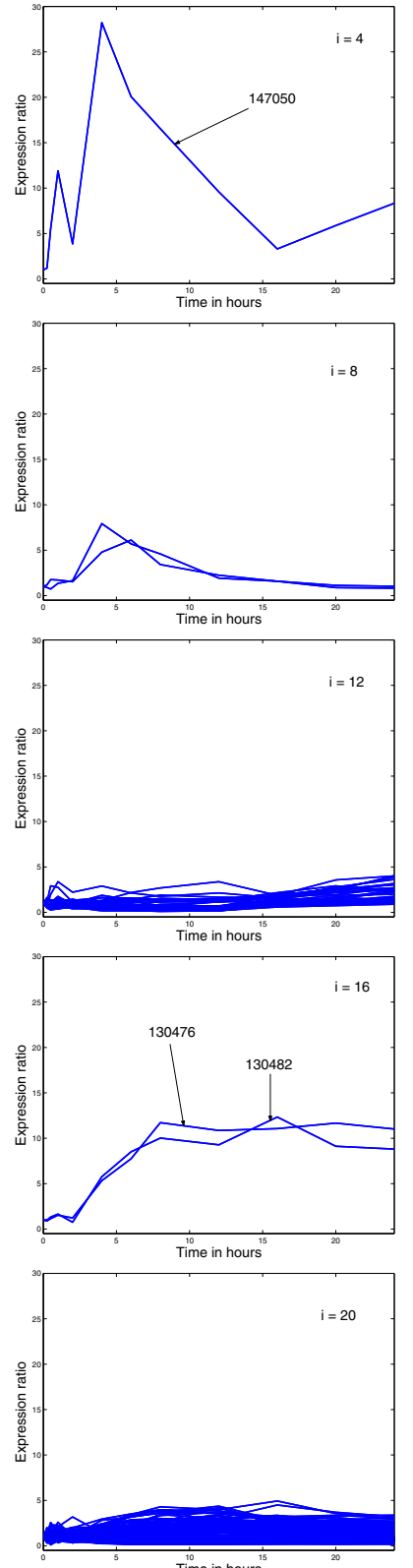

${ }^{10}{ }^{10}$ ime in hours

Figure 3: Different clusters obtained using PAAC on the 517 gene temporal expression profiles, where $k=21$. 

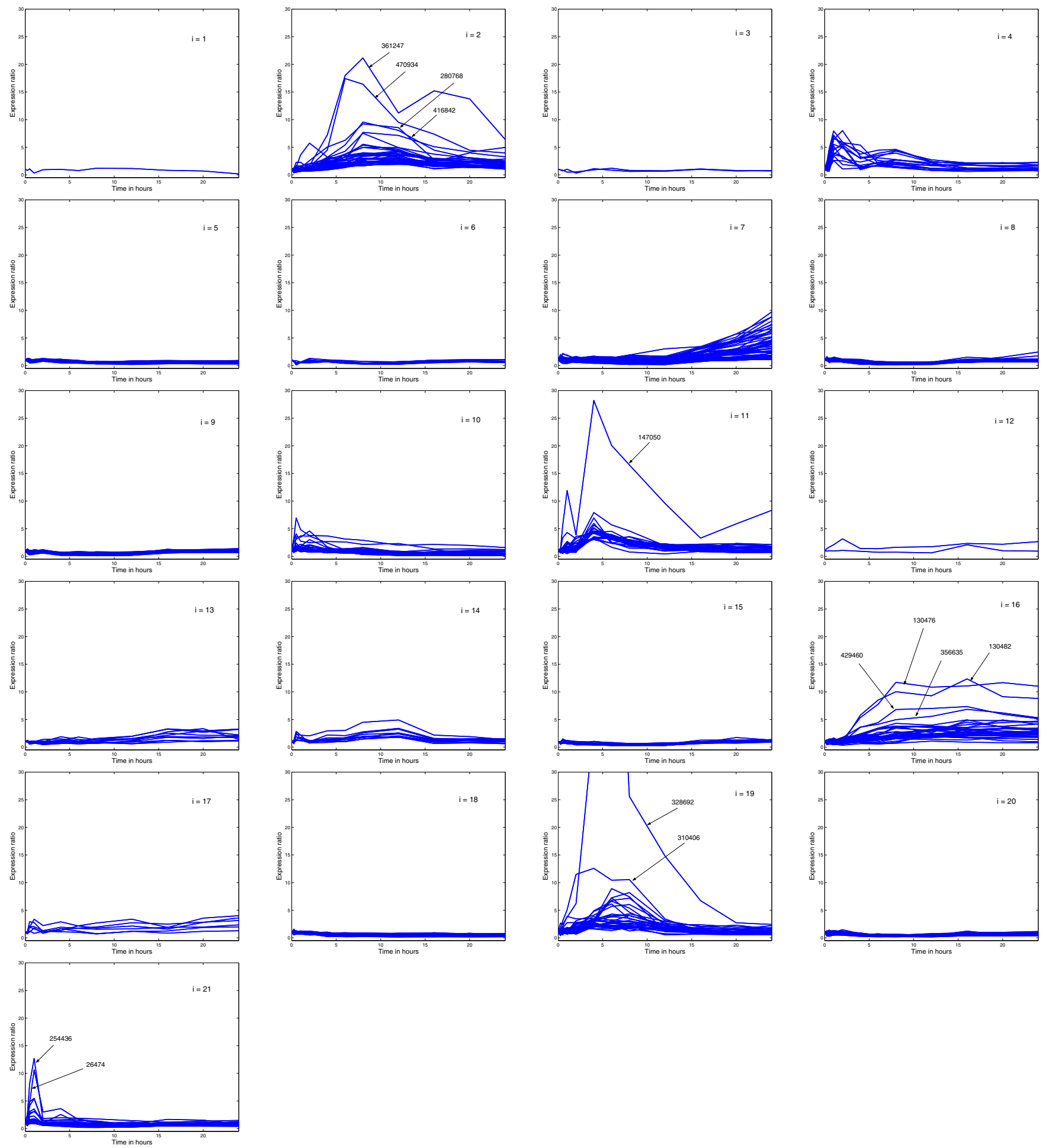

Figure 4: Different clusters obtained using the Pearson correlation distance on the 517 gene temporal expression profiles, where $k=21$. 

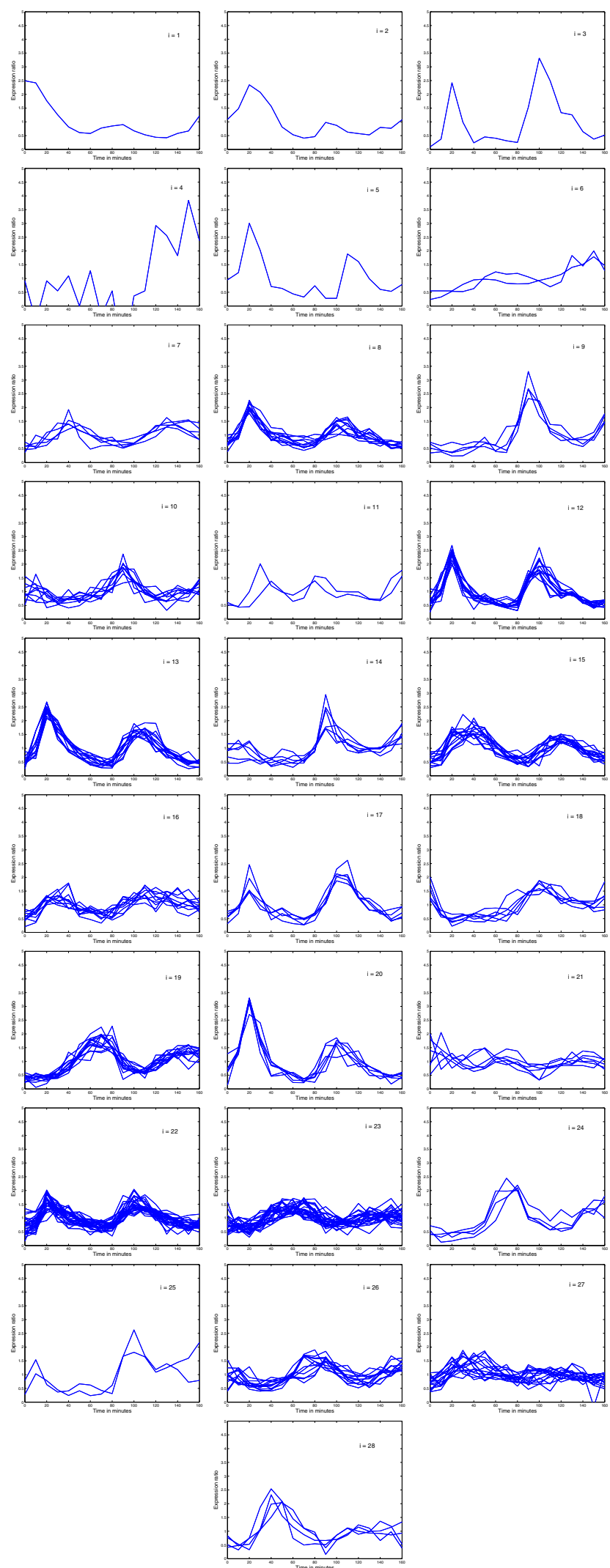

Figure 5: Different clusters obtained using PAAC on the 221 gene temporal expression profiles from Table 1 of [13], where $k=28$.
[7] L. Heyer, S. Kruglyak, and S. Yooseph. Exploring expression data: identification and analysis of coexpressed genes. Genome Res., 9:1106-1115, 1999.

[8] V. Iyer, M. Eisen, D. Ross, G. Schuler, T. Moore, J. Lee, J. Trent, L. Staudt, Jr. J. Hudson, and M. Boguski. The transcriptional program in the response of human fibroblasts to serum. Science, 283:83-87, 1999.

[9] U. Maulik and S. Bandyopadhyay. Performance Evaluation of Some Clustering Algorithms and Validity Indices. IEEE Transactions on Pattern Analysis and Machine Intelligence, 24(12):1650-1654, 2002.

[10] S. Peddada, E. Lobenhofer, L. Li, C. Afshari, C. Weinberg, and D. Umbach. Gene selection and clustering for time-course and dose-response microarray experiments using order-restricted inference. Bioinformatics, 19(7):834-841, 2003.

[11] G. Sherlock. Analysis of large-scale gene expression data. Curr. Opin. Immunol., 12:201-205, 2000.

[12] A. Bari and L. Rueda. A New Profile Alignment Method for Clustering Gene Expression Data. In proceedings of 19th Conference of the Canadian Society for Computational Studies of Intelligence (AI 2006), Quebec City, LNCS, Vol 4013:86-97, Springer, 2006.

[13] R. J. Cho, M.J.Campbell, E. A. Winzeler, L. Steinmetz, A. Conway, L. Wodicka, T. G. Wolfsberg, A. E. Gabrielian, D. Landsman, D. J. Lockhart, R. W. Davis. A genome-wide transcriptional analysis of the mitotic cell cycle. Molecular Cell, 2(1):65-73, 1998. 


\begin{tabular}{|c|c|c|c|c|c|c|c|c|}
\hline PAAC & Gene & Phase & PAAC & Gene & Phase & PAAC & Gene & Phase \\
\hline 1 & YBR067c/TIP1 & Early $G_{1}$ & 15 & YEL061c/CIN8 & $S$ & 22 & YLL002w/ & Late $G_{1}$ \\
\hline 2 & YGL055W/OLE1 & Early $G_{1}$ & 15 & YGR140W/CBF2 & $S$ & 22 & YLR457C/NBP1 & Late $G_{1}$ \\
\hline 3 & YDL227C/HO & Late $G_{1}$ & 15 & YHR172W/ & $S$ & 22 & YPL057C/SUR1 & Late $G_{1}$ \\
\hline 4 & YAL001C/TFC3 & $S$ & 15 & YLR045c/STU2 & $S$ & 22 & YPL124W/NIP29 & Late $G_{1}$ \\
\hline 5 & YJL115W/ASF1 & $S$ & 15 & YNL126W/ & $S$ & 22 & YBR275c/RIF1 & \\
\hline 6 & YDL198C/SHM1 & $G_{2}$ & 15 & YPR $141 \mathrm{C} / \mathrm{KAR} 3$ & $S$ & 22 & YCR065w/HCM1 & $S$ \\
\hline 6 & YDR146c/SWI5 & $M$ & 15 & YJR006W/HUS2 & $S$ & 22 & YDL197C/ASF2 & $S$ \\
\hline 7 & YKL049C/CSE4 & $G_{2}$ & 15 & YER001w/MNN1 & $S$ & 22 & YKL127W/PGM1 & $S$ \\
\hline 7 & YKL048C/ELM1 & $G_{2}$ & 15 & YER003c/PMI40 & $S$ & 22 & YDL095W/PMT1 & $S$ \\
\hline 7 & YER069w/"ARG5,6" & $G_{2}$ & 16 & YNL225C/ & Late $G_{1}$ & 23 & YLR210W/CLB4 & $S$ \\
\hline 7 & YJR112W/NNF1 & $G_{2}$ & 16 & YDR224c/HTB1 & Late $G_{1}$ & 23 & YDR150w/NUM1 & $M$ \\
\hline 7 & YPR167C/MET16 & $M$ & 16 & YDR $225 \mathrm{w} / \mathrm{HTA} 1$ & Late $G_{1}$ & 23 & YMR198W/CIK1 & $S$ \\
\hline 8 & YFL008W/SMC1 & Late $G_{1}$ & 16 & YGL200C/EMP24 & Late $G_{1}$ & 23 & YBL052c/ & $S$ \\
\hline 8 & YMR078C/CHL12 & Late $G_{1}$ & 16 & YNL $272 \mathrm{C} / \mathrm{SEC} 2$ & Late $G_{1}$ & 23 & YIL126W/STH1 & $S$ \\
\hline 8 & YPL209C/IPL1 & Late $G_{1}$ & 16 & YDR488c/PAC11 & & 23 & YCR035c/ & $S$ \\
\hline 8 & YGR152C/RSR1 & Late $G_{1}$ & 16 & YBL002w/HTB2 & $S$ & 23 & YIL050W/ & $G_{2}$ \\
\hline 8 & YIL159W/ & Late $G_{1}$ & 16 & YBL003c/HTA2 & $S$ & 23 & YBL097w/ & $G_{2}$ \\
\hline 8 & YNL233W/ & Late $G_{1}$ & 16 & YKL067W/YNK1 & $S$ & 23 & YJL099W/CHS6 & $G_{2}$ \\
\hline 8 & YKL045W/PRI2 & Late $G_{1}$ & 16 & YER118c/SSU81 & $S$ & 23 & YJR076C/CDC11 & $G_{2}$ \\
\hline 8 & YNL312W/RFA2 & Late $G_{1}$ & 17 & YJL074C/SMC3 & Late $G_{1}$ & 23 & YCR084c/TUP1 & $G_{2}$ \\
\hline 8 & YML061C/PIF 1 & Late $G_{1}$ & 17 & YGR041W/ & Late $G_{1}$ & 23 & YGL255W/ZRT1 & $G_{2}$ \\
\hline 8 & YLR233C/EST1 & Late $G_{1}$ & 17 & YNL082W/PMS1 & Late $G_{1}$ & 23 & YJL137c/GLG2 & $G_{2}$ \\
\hline 8 & YOR026W/BUB3 & $S$ & 17 & YOL090W/MSH2 & Late $G_{1}$ & 23 & YCR073c/ & $G_{2}$ \\
\hline 9 & YDL179w/ & Early $G_{1}$ & 17 & YHR153c/SPO16 & Late $G_{1}$ & 23 & YDR389w/SAC7 & $G_{2}$ \\
\hline 9 & YLR079w/SIC1 & Early $G_{1}$ & 18 & YCR005c/CIT2 & Early $G_{1}$ & 23 & YKL068W/NUP100 & $G_{2}$ \\
\hline 9 & YJL157C/FAR1 & Early $G_{1}$ & 18 & YCL040w/GLK1 & Early $G_{1}$ & 23 & YGR092W/DBF2 & $M$ \\
\hline 9 & YKL185W/ASH1 & Early $G_{1}$ & 18 & YLR258W/GSY2 & Early $G_{1}$ & 23 & YOR058C/ASE1 & $M$ \\
\hline 10 & YJL194W/CDC6 & Early $G_{1}$ & 18 & YNL173C/ & Late $G_{1}$ & 23 & YPL242C/ & $M$ \\
\hline 10 & YLR274W/CDC46 & Early $G_{1}$ & 18 & YOR $317 \mathrm{~W} / \mathrm{FAA} 1$ & Late $G_{1}$ & 23 & YCL037c/SRO9 & $M$ \\
\hline 10 & YPR019W/CDC54 & Early $G_{1}$ & 18 & YNL073W/MSK1 & $S$ & 23 & YKL130C/ & $M$ \\
\hline 10 & YHR005c/GPA1 & Early $G_{1}$ & 19 & YIL106W/MOB1 & $G_{2}$ & 23 & YNL053W/MSG5 & $M$ \\
\hline 10 & YGR183C/QCR9 & Early $G_{1}$ & 19 & YCL014w/BUD3 & $G_{2}$ & 23 & YIL162W/SUC2 & $M$ \\
\hline 10 & YLR273C/PIG1 & Early $G_{1}$ & 19 & YGR108W/CLB1 & $M$ & 23 & YDL048c/STP4 & $M$ \\
\hline 10 & YLL040c/ & Early $G_{1}$ & 19 & YPR119W/ & $M$ & 23 & YHR152w/SPO12 & $M$ \\
\hline 10 & YHR038W/ & Late $G_{1}$ & 19 & YBR138c/ & $M$ & 23 & YKL129C/MYO3 & $M$ \\
\hline 10 & YAL040C/CLN3 & $M$ & 19 & YHR023w/MYO1 & $M$ & 24 & YPL058C/PDR12 & Early $G_{1}$ \\
\hline 11 & YDR277c/MTH1 & $S$ & 19 & YOL069W/NUF2 & $M$ & 24 & YBR038w/CHS2 & $G_{2}$ \\
\hline 11 & YML091C/RPM2 & $S$ & 19 & YJR092W/BUD4 & $M$ & 24 & YGL116W/CDC20 & $M$ \\
\hline 12 & YDL127w/PCL2 & Late $G_{1}$ & 19 & YLR353W/BUD8 & $M$ & 24 & YGR143W/SKN1 & $M$ \\
\hline 12 & YPR120C/ & Late $G_{1}$ & 19 & YMR001C/CDC5 & $M$ & 25 & YLR286C/CTS1 & Late $G_{1}$ \\
\hline 12 & YDL003W/RHC21 & Late $G_{1}$ & 19 & YGL021W/ALK1 & $M$ & 25 & YGL089C/MF(alpha)2 & Late $G_{1}$ \\
\hline 12 & YAR007C/RFA1 & Late $G_{1}$ & 19 & YLR131c/ACE2 & $M$ & 26 & YBR200w/BEM1 & Early $G_{1}$ \\
\hline 12 & YBL035c/POL12 & Late $G_{1}$ & 19 & YOR025W/HST3 & $M$ & 26 & YBL023c/MCM2 & Early $G_{1}$ \\
\hline 12 & YBR088c/POL30 & Late $G_{1}$ & 20 & YGR109C/CLB6 & Late $G_{1}$ & 26 & YBR202w/CDC47 & Early $G_{1}$ \\
\hline 12 & YDL164C/CDC9 & Late $G_{1}$ & 20 & YNL289W/PCL1 & Late $G_{1}$ & 26 & YEL032w/MCM3 & Early $G_{1}$ \\
\hline 12 & YML102W/ & Late $G_{1}$ & 20 & YLR313C/ & Late $G_{1}$ & 26 & YLR395C/COX8 & Early $G_{1}$ \\
\hline 12 & YPR175W/DPB2 & Late $G_{1}$ & 20 & YPR018W/RLF2 & Late $G_{1}$ & 26 & YMR256c/COX7 & Early $G_{1}$ \\
\hline 12 & YDR097C/ & Late $G_{1}$ & 20 & YPL153C/SPK1 & Late $G_{1}$ & 26 & $\mathrm{R} 281 \mathrm{~W} / \mathrm{YOR} 1$ & Early $G_{1}$ \\
\hline 12 & YLR032w/RAD5 & Late $G_{1}$ & 20 & YBR070c/ & Late $G_{1}$ & 26 & YOR316C/COT1 & Late $G_{1}$ \\
\hline 12 & YML027W/YOX1 & Late $G_{1}$ & 21 & YJR159W/SOR1 & $G_{2}$ & 26 & YCR042c/TSM1 & $M$ \\
\hline 12 & YMR179W/SPT21 & Late $G_{1}$ & 21 & YBR104w/YMC2 & $G_{2}$ & 26 & YOR229W/ & $M$ \\
\hline 13 & YJL187C/SWE1 & Late $G_{1}$ & 21 & YLR014c/PPR1 & $G_{2}$ & 26 & YDL138W/RGT2 & $M$ \\
\hline 13 & YPL256C/CLN2 & Late $G_{1}$ & 21 & YOR274W/MOD5 & $G_{2}$ & 26 & YIL167W/ & $M$ \\
\hline 13 & YMR076C/PDS5 & Late $G_{1}$ & 21 & YDR 464w/SPP41 & $G_{2}$ & 27 & YNR016C/ACC1 & Early $G_{1}$ \\
\hline 13 & YER070w/RNR1 & Late $G_{1}$ & 21 & YLL046c/RNP1 & $G_{2}$ & 27 & YBR $160 \mathrm{w} / \mathrm{CDC} 28$ & Late $G_{1}$ \\
\hline 13 & YLR103c/CDC45 & Late $G_{1}$ & 22 & YER111c/SWI4 & Early $G_{1}$ & 27 & YBR $252 \mathrm{w} / \mathrm{DUT} 1$ & Late $G_{1}$ \\
\hline 13 & YNL102W/CDC17 & Late $G_{1}$ & 22 & YOR373W/NUD1 & Early $G_{1}$ & 27 & YBR278w/DPB3 & Late $G_{1}$ \\
\hline 13 & YOR074C/CDC21 & Late $G_{1}$ & 22 & YKL092C/BUD2 & Early $G_{1}$ & 27 & YDR297w/SUR2 & Late $G_{1}$ \\
\hline 13 & YKL113C/RAD27 & Late $G_{1}$ & 22 & YMR199W/CLN1 & Late $G_{1}$ & 27 & YDL155W/CLB3 & $S$ \\
\hline 13 & YLR383W/ & Late $G_{1}$ & 22 & YKL042W/ & Late $G_{1}$ & 27 & YFR037C/ & $S$ \\
\hline 13 & YML060W/OGG1 & Late $G_{1}$ & 22 & YLR212C/TUB4 & Late $G_{1}$ & 27 & YPL016W/SWI1 & $S$ \\
\hline 13 & YIL140W/SRO4 & $S$ & 22 & YPL241C/CIN2 & Late $G_{1}$ & 27 & YDL093W/PMT5 & $S$ \\
\hline 13 & YAR008W/ & $S$ & 22 & YDR507c/GIN4 & Late $G_{1}$ & 27 & YKR001C/SPO15 & $S$ \\
\hline 14 & YDL181W/INH1 & Early $G_{1}$ & 22 & YGL027C/CWH41 & Late $G_{1}$ & 27 & YER016w/BIM1 & $S$ \\
\hline 14 & YML110C/ & Early $G_{1}$ & 22 & YJL173C/RFA3 & Late $G_{1}$ & 27 & YER017c/AFG3 & $S$ \\
\hline 14 & YIL009W/FAA3 & Early $G_{1}$ & 22 & YNL262W/POL2 & Late $G_{1}$ & 27 & YPR $111 \mathrm{~W} / \mathrm{DBF} 20$ & $G_{2}$ \\
\hline 14 & YBR083w/TEC 1 & Early $G_{1}$ & 22 & YDL101C/DUN1 & Late $G_{1}$ & 27 & YOR188W/MSB1 & $G_{2}$ \\
\hline 14 & YPL $187 \mathrm{~W} / \mathrm{MF}$ (alpha) 1 & Late $G_{1}$ & 22 & YLR234W/TOP3 & Late $G_{1}$ & 27 & YJL092W/HPR5 & $G_{2}$ \\
\hline 14 & YJR148W/TWT2 & Late $G_{1}$ & 22 & YML021C/UNG1 & Late $G_{1}$ & 27 & YKL032C/IXR1 & $G_{2}$ \\
\hline 15 & YPL127C/HHO1 & Late $G_{1}$ & 22 & YLR382C/NAM2 & Late $G_{1}$ & 28 & YMR190C/SGS1 & $S$ \\
\hline 15 & YLL021w/SPA2 & Late $G_{1}$ & 22 & YJL196C/ & Late $G_{1}$ & 28 & YIR017C/MET28 & $S$ \\
\hline 15 & YBL063w/KIP1 & $S$ & 22 & YBR073w/RDH54 & Late $G_{1}$ & 28 & YHR086w/NAM8 & $S$ \\
\hline 15 & YDR113c/PDS1 & $S$ & 22 & YKL101W/HSL1 & Late $G_{1}$ & 28 & YJR137C/ & $S$ \\
\hline 15 & YDR356w/NUF1 & $S$ & 22 & YKL165C/ & Late $G_{1}$ & & & \\
\hline
\end{tabular}

Table 1: Genes from Table 1 of [13], clustered using PAAC, where $k=28$. 